\title{
Predicted Performance of an Induction Linac Driven FEL Amplifier Designed for Radar Applications at $94 \mathbf{G H z}$
}

\author{
M. Caplan
}

This paper was prepared for submittal to the Thirteenth International Free-Electron Laser Conference August 25-30, 1991, Santa Fe, New Mexico

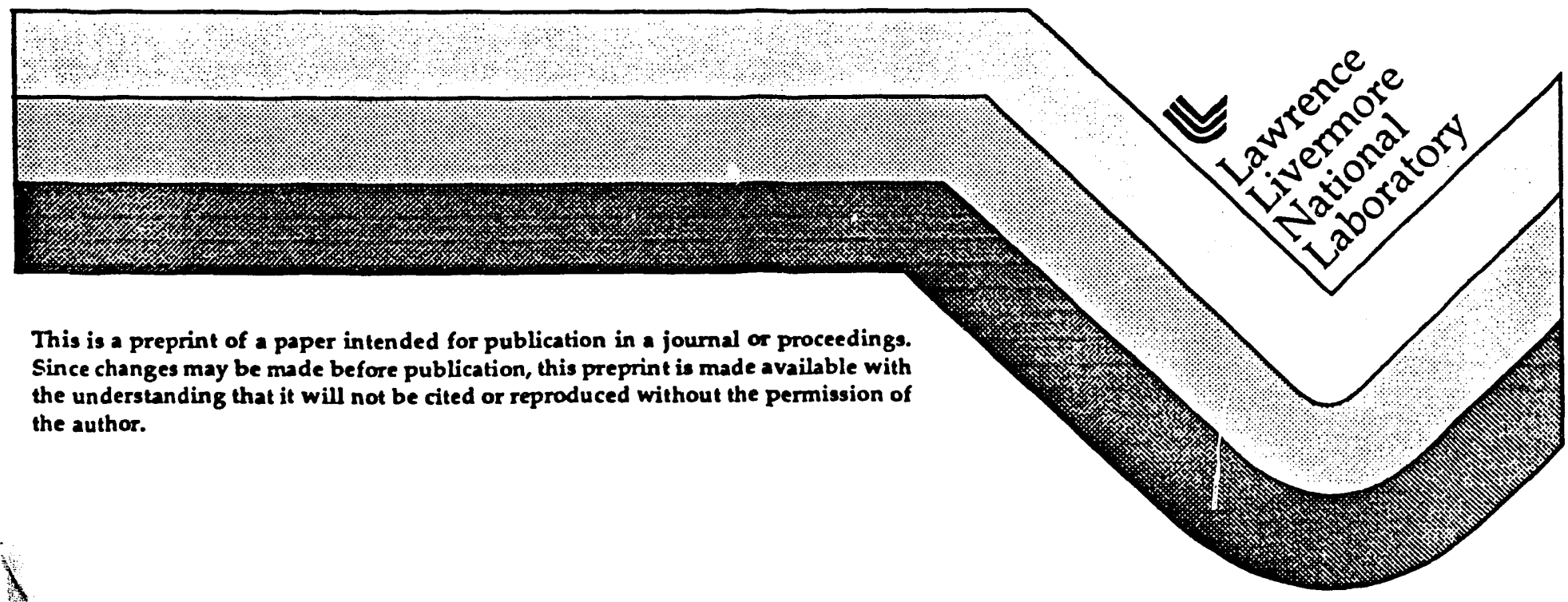




\section{DISCLAIMER}

This document was prepared as an sccoont of work sponsoned by an asency of the United States Government. Neither the United States Government wor the University of California nor any of their employees, makes any warranty, express or implied, or essumes any legal liability or responsibility for the accuracy, completeness, or aseforness of any information, apparatus, product, or process disclosed, or represents that its use would not infringe privately owned rights. Reference herein to any specific commercial prodects, process, or service by trade anme, trademark, manufacturer, or otherwise, does not mecesenrily constitute or imply its endorsement, reconmendation. or favoring by the United States Covernment or the Univensity of California. The views and opinions of authors expreased herein do wot mecesearily state or reflect those of the United States Government or the Univeraity of Callforain, and shall sot be ased for advertising or prodect eadorsement perposes. 
Predicted Performance of an Induction Linac Driven

FEL Amplifier Designed for Radar Applications at $94 \mathrm{GHz}^{*}$

\author{
M. Caplan \\ Lawrence Livermore National Laboratory \\ Livermore, CA 94550
}

\begin{abstract}
The saturated performance of an Induction Linac Driven FEL operating at $94 \mathrm{GHz}$ with peak microwave powers in excess of $2 \mathrm{GW}$ is evaluated using a self-consistent $3 \mathrm{D}$ waveguide mode-particle interaction code. The code includes the effect of AC longitudinal space charge and DC transverse space charge with the resulting prediction that operation is not possible below 2.5 $\mathrm{MeV}$ with a $2 \mathrm{kA}$ beam and $10 \mathrm{~cm}$ wiggler. A design based around a $4 \mathrm{MeV}$ beam predicts peak power in excess of $2.4 \mathrm{GW}$ over a $10 \%$ bandwidth with output phase varying linear with frequency.
\end{abstract}

\title{
1. Introduction
}

There is interest in developing a $94 \mathrm{GHz}$ FEL amplifier as the microwave source for an imaging radar. The radar is required to operate at a power of 2 GW peak, $5 \mathrm{MW}$ average with a bandwidth of at least $5 \%$. The beam to power the FEL is provided by an induction linear accelerator operating in the range 2-5 $\mathrm{MeV}$ and 1-2 $\mathrm{kA}$ with $1 \mu \mathrm{sec}$ pulses. The requirement for high average power leads to the choice of a $10 \mathrm{~cm}$ period linear polarized wiggler with curved pole pieces for focusing and capable of generating up to 4 kilogauss transverse field. It is practical from the point of view of accelerator design to operate at lower voltages and higher currents consistent with high microwave conversion efficiency. In order to determine a reasonable operating voltage, it is important to include both AC and DC space charge effects in the FEL computer models since these effects can greatly degrade

*This work was performed under the auspices of the U.S. Department of Energy by Lawrence Livermore National Laboratory under concract W-7405ENG-48. 
performance at lower voltages. A 3D self-consistent microwave FEL code has been developed in order to design and predict the performance of an FEL satisfying the above radar requirements. In Sec. 2, a brief description of the computer model is given. Section 3. shows the effects of AC and DC space charge on determining the design. Finally, in Sec. 4. a $94 \mathrm{GHz}$ FEL design is presented with predictions of power, mode purity and phase versus frequency.

\section{Computer Model}

A self-consistent 3D microwave FEL code has been developed with the following features:

(i) Non-wiggle averaged exact 3D particle orbits.

(ii) Initial particles set up to fill a 4 volume in phase space uniformly corresponding to a specified beam emittance. Realistic initial focusing and entrance conditions can be modelled. A spread in gamma due to space charge depression is included.

(iii) Fields are expanded in a complete set of TE and TM modes in arbitrary waveguide geometry.

(iv) AC space charge is included up to 5 th harmonic in $\left[\omega t-\mathrm{kv}_{\mathrm{z}}\right]$. This is rigorously derived by summing up the $\mathrm{E}_{\mathbf{z}}$ fields of all nonresonant TM modes.

(v) DC space charge forces are included by solving for the DC radial electric field and potential in the center of mass frame using gausses law.

(vi) External 3D magnetic fields are those of a curved pole piece tapered wiggler of straight pole piece with quadrupole focusing.

The particle equations of motion for the transverse positions, momenta, phase $\psi=\left(\omega t-k_{1} z\right)$ and gamma $(\gamma)$ as functions of $z$ are given by:

$$
\begin{aligned}
& \frac{d x_{p}}{d z}=\frac{U_{x}^{p}}{U q}, \quad \frac{d y_{p}}{d z}=\frac{U_{y}^{p}}{U q}, \quad \frac{d \psi_{p}}{d z}=\frac{\omega \gamma_{p}}{U_{z}^{p} c}-k_{I} \\
& \frac{d U p}{d z}=-\frac{e E_{x}}{m c^{2}} \cdot \frac{\gamma_{p}}{U q}-\frac{U_{y}^{\rho}}{U U_{z}} \frac{e B_{z}}{m c^{2}}+\frac{e B_{y}}{m c^{2}} \\
& \frac{d U p}{d z}=-\frac{e E_{y}}{m c^{2}} \frac{\gamma_{p}}{U \rho}-\frac{e B_{x}}{m c^{2}}+\frac{U_{x}^{\rho}}{U q} \frac{e B_{z}}{m c^{2}}
\end{aligned}
$$




$$
\frac{d \gamma_{p}^{*}}{d z}=-\frac{\theta}{m c^{2}} \frac{\vec{U}^{p} \cdot \vec{E} \text { (microwave) }}{U \underline{u}}
$$$$
\gamma_{p}=\dot{\gamma_{p}}-\frac{e|\Phi|_{p}}{m c^{2}} \quad \text { where: } \psi p \text { is particle phase }=\left(\omega t-k_{I} z\right)
$$$$
\Phi_{\mathrm{P}} \text { is DC space charge potential }
$$

$$
U U_{2}=\sqrt{\gamma_{p}^{2}-1-\left(U p_{x}\right)^{2}-\left(U_{y}\right)^{2}}
$$

The generalized field equations for arbitrary waveguide geometry are given in terms of the complex wave amplitude $V(z)$ by:

$$
\begin{aligned}
\vec{E} & =\sum_{i} R_{\theta}\left\{V(z) e^{j \omega t-j k_{z}^{i} z}\right\} \sqrt{Z_{i}} \vec{\varepsilon}_{i}(x, y) \\
& +\hat{z} \sum_{i} I_{m}\{\underbrace{\left.V_{i}(z) e^{j \omega t-j k i} z_{z}^{i}\right\} k_{d}^{i} d\left(k_{\theta} \sqrt{Z_{i}}\right)}_{T M \text { modes }} \cdot \dot{\varepsilon}_{z}^{j}(x, y)+\vec{E} \text { (space charge) }
\end{aligned}
$$

$$
\begin{aligned}
\vec{B} & =\sum_{i} R_{\theta}\left\{V(z) e^{j \omega t-j k_{z}^{i} z}\right\} \frac{1}{\sqrt{Z_{i}}} \cdot\left(\hat{z} \times \vec{\varepsilon}_{i}(x, y)\right) \\
& +\hat{z} \sum_{i} \underbrace{I_{m}\left\{V_{i}(z) e^{j \omega t-j k_{z}^{i} z}\right\} k_{e}^{i}\left(\sqrt{Z_{i}} / k_{0}\right)}_{\text {TE modes }} \cdot b_{z}^{i}(x, y)
\end{aligned}
$$

$$
\begin{aligned}
\frac{d V_{i}(z)}{d z} & =\frac{2}{N_{p}} \exp \left(-j \Delta k_{i} z\right) \cdot \frac{2 \pi I_{0} \sqrt{Z_{i}}}{c} \sum_{p} e^{-j \psi_{p}} \frac{\vec{U}_{1}^{p}}{U q} \cdot \vec{\varepsilon}_{l}\left(x_{p}, y_{p}\right) \\
& +\frac{2}{N_{p}} \exp \left(-j \Delta k_{i} z\right) \cdot \frac{2 \pi I_{0} k_{c}^{i}}{k_{o} \sqrt{Z_{i}}} \sum_{p} e^{-j \psi_{p}} e^{i}\left(x_{p}, y_{p}\right) \mid \quad T M \text { modes only }
\end{aligned}
$$

Power $=\frac{c}{4 \pi} \frac{\left|V_{i}\right|^{2}}{2} \quad \varepsilon(x y)=$ normalized waveguide transverse functions 
The evaluation of the AC space charge field is obtained by analytically summing the axial components of the TM modes and gives the same expression as that used in the "FRED" code.[1] The DC space charge fields are obtained from gausses law. These fields are given explicitly as:

AC Axial E field

$$
\begin{aligned}
& E_{z}=\sum_{m} \frac{4 \pi j \rho_{m}}{m\left(k+k_{w}\right)} e^{j m\left[\omega t-\left(k+k_{m} k\right]\right.} \cdot\left[1-\frac{4}{\left(m g r_{b}\right)}-\left(\frac{r}{r_{b}}\right)^{2}-f_{m} I_{d}(m g r)\right] \\
& f_{m}=-2\left[I_{0}\left((m g a) K_{2}\left(m g r_{0}\right)-K_{0}(m g a) I_{2}\left(m g r_{b}\right)\right]\right. \\
& g=\frac{\left(k+k_{w}^{\prime}\right) a}{\gamma} \\
& \text { a }=\text { guide radius } \\
& \mathrm{r}_{\mathrm{b}}=\text { beam radius } \\
& \rho_{m}=-\frac{4 I_{0}}{\pi r_{b}^{2} C} \frac{e^{j m k_{w} z}}{N_{p}} \sum_{p} e^{-i m \psi_{p}}=m \text { th harmonic of charge density }
\end{aligned}
$$

DC space charge potential

$$
\frac{e \Phi}{m c^{2}}=-\frac{2 e I_{o}}{m c^{3}} \ln \left(\frac{a}{r_{b}}\right)-\frac{2 e I_{o}}{m c^{3}} \int_{r}^{r_{b}} \frac{N(r)}{N T r} d r
$$

\section{Effect of AC and DC. Space Charge on FEL Design}

It is desirable for practical reasons to lower the accelerator voltage while still maintaining efficiency and frequency. The physics of FEL interaction indicated that the "bucket height" decreases with decreasing voltage and increasing $A C$ space charge assuming fixed frequency. [2] If the spread in $\gamma$ due to DC space charge depression is much greater than the bucket height, FEL performance is severely reduced. This is expressed mathematically by: 


$$
\begin{aligned}
(\Delta \gamma)_{\text {Bucket }}=\left\{\left(\frac{4 e \gamma}{m c^{2} k_{W}}\right)\left[E_{R F} \sqrt{\frac{c k_{W}}{\omega}-\frac{1}{2 \gamma^{2}}}-E_{S p}\right]\right\}^{1 / 2}>.088 I_{0}(k i l o a m p s) \\
\text { spread due to } D C \\
\text { space charge }
\end{aligned}
$$

where $E_{\mathrm{sp}}=\mathrm{AC}$ space charge field $=4 \mu \mathrm{c} \rho_{\mathrm{m}} / \omega$

$$
\begin{aligned}
& E_{R F}=R F \text { electric field } \\
& I_{0}=\text { Beam current }
\end{aligned}
$$

Computer simulations were performed at various voltages and the power optimized as illustrated in Fig. 1. FEL performance is severely degraded at $2 \mathrm{kA}$ as one lowers the voltage to $2.5 \mathrm{MeV}$. Figure 2 shows that this is due to the combined effects of both $A C$ and DC space charge fields. The AC space charge fields reduce the bucket height decreasing gain and bunching. The effect of DC space charge at $2 \mathrm{kA}$ results in sufficient spread in $\gamma$ so that the reduced bucket no longer can contain the electrons. In order to develop a conservative robust design where space charge effects do not present problems, the voltage was chosen to be $4 \mathrm{MeV}$. Table I gives the basic design parameters for the proposed $94 \mathrm{GHz}$ FEL.

\section{Performance Predictions for FEL Design}

Simulations were done using the parameters of Table I to evaluate the amplifier characteristics at saturation at 1000 watts drive. Typical simulation results are shown Fig. 3. The first 1.9 meters is a linear gain region with the eigenmode made up of $80 \% \mathrm{TE}_{01}$ mode and $20 \%$ in $\mathrm{TE}_{21}, \mathrm{TE}_{41}$, and $\mathrm{TM}_{21}$. The remaining 2.1 meters consists of a linear taper of 3\% decrease per wiggler period. The final output power is almost entirely in the $\mathrm{TE}_{01}$ mode. Figure 4 shows that the effect of the DC space charge is to cause the beam to expand $40 \%$ more in the tapered region than it would otherwise. This puts extra restriction on how much tapering can be done before the beam gets near the waveguide wall and thus restricts the maximum achievable efficiency. FEL performance is summarized in Fig. 5 which shows power, phase and mode purity as a function of frequency. One observes that there is a frequency range 
of almost $10 \mathrm{GHz}$ where the phase varies linear with frequency and the power is within $1 \mathrm{~dB}$ of maximum with mode purity greater than $96 \%$.

\section{Conclusions}

A conservative design has been developed for a $94 \mathrm{GHz}$ FEL amplifier for radar applications demanding high average power. The basic performance characteristics are:

$$
\begin{array}{ll}
\text { peak power } & \geq 2.4 \mathrm{GW} \\
\text { average power } & 5 \mathrm{MW} \\
1 \mathrm{~dB} \text { bandwidth } & 10 \% \\
\text { phase linearity bandwidth } & 10 \% \\
\text { mode purity over bandwith } & \geq 96 \%
\end{array}
$$

\section{References}

[1] E.T. Scharlemann, W.M. Fawley, B. Anderson, and T.J. Orzechowski, "Comparison of the Livermore Microwave FEL Results at ELF with 2D Numerical Simulation," Nuclear Instruments and Methods in Physics, Research A250 (1986) 150-158.

[2] V.K. Neil, "Effects of Longitudinal Electric Self-Field on Acceptable Energy Spread in a Free Electron Laser," UCID-179851, LLNL, November 27, 1978. 
Figure 1 Maximum power out versus beam voltage. Current fixed at $2 \mathrm{kA}$. Frequency fixed at $94 \mathrm{GHz}$. Linear field tapered down to $20 \%$ of maximum wiggler field.

Figure 2a Bunch parameter $\left\langle\mathrm{e}^{\mathrm{i} \psi}\right\rangle$ versus distance with and without $A C$ and DC space charge. Voltage $=2.5 \mathrm{MeV} \quad$ Current $=2 \mathrm{kA}$

Figure $2 b$ Gain versus distance with and without $A C$ and DC space charge. Voltage $=2.5 \mathrm{MeV} \quad$ Current $=2 \mathrm{kA}$

Figure 3 Power and mode purity $\left(\% \mathrm{TE}_{01}\right)$ vs distance with magnetic taper. Voltage $=4 \mathrm{MeV} \quad$ Current $=2 \mathrm{kA}$

Figure 4 Effect of DC space charge on beam expansion in linear taper region. Voltage $=4 \mathrm{MeV} \quad$ Current $=2 \mathrm{kA}$

Figure 5 Power, phase and mode purity vs frequency at saturation. Drive power $=1000$ watts. 


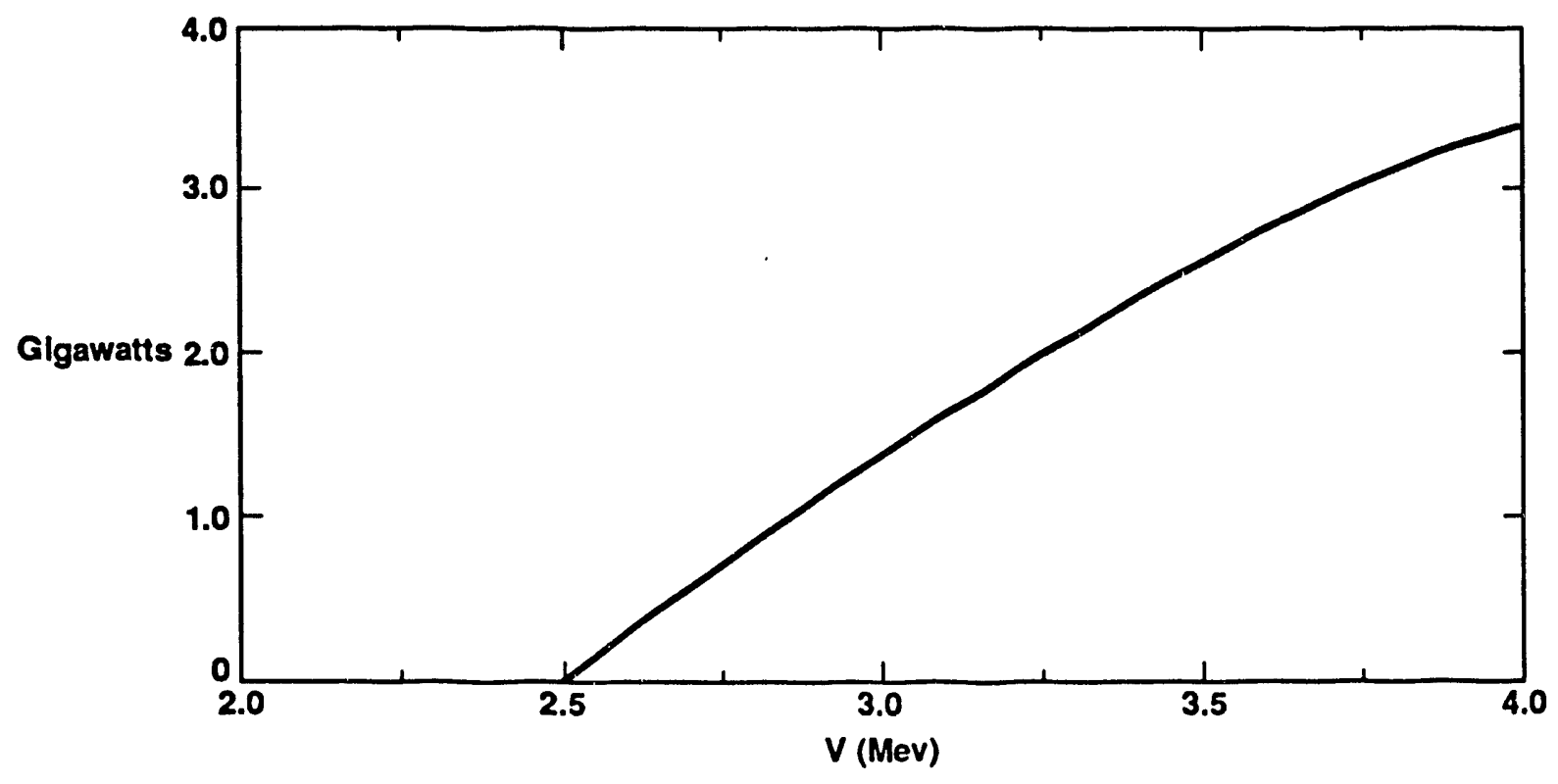

Figure 1 

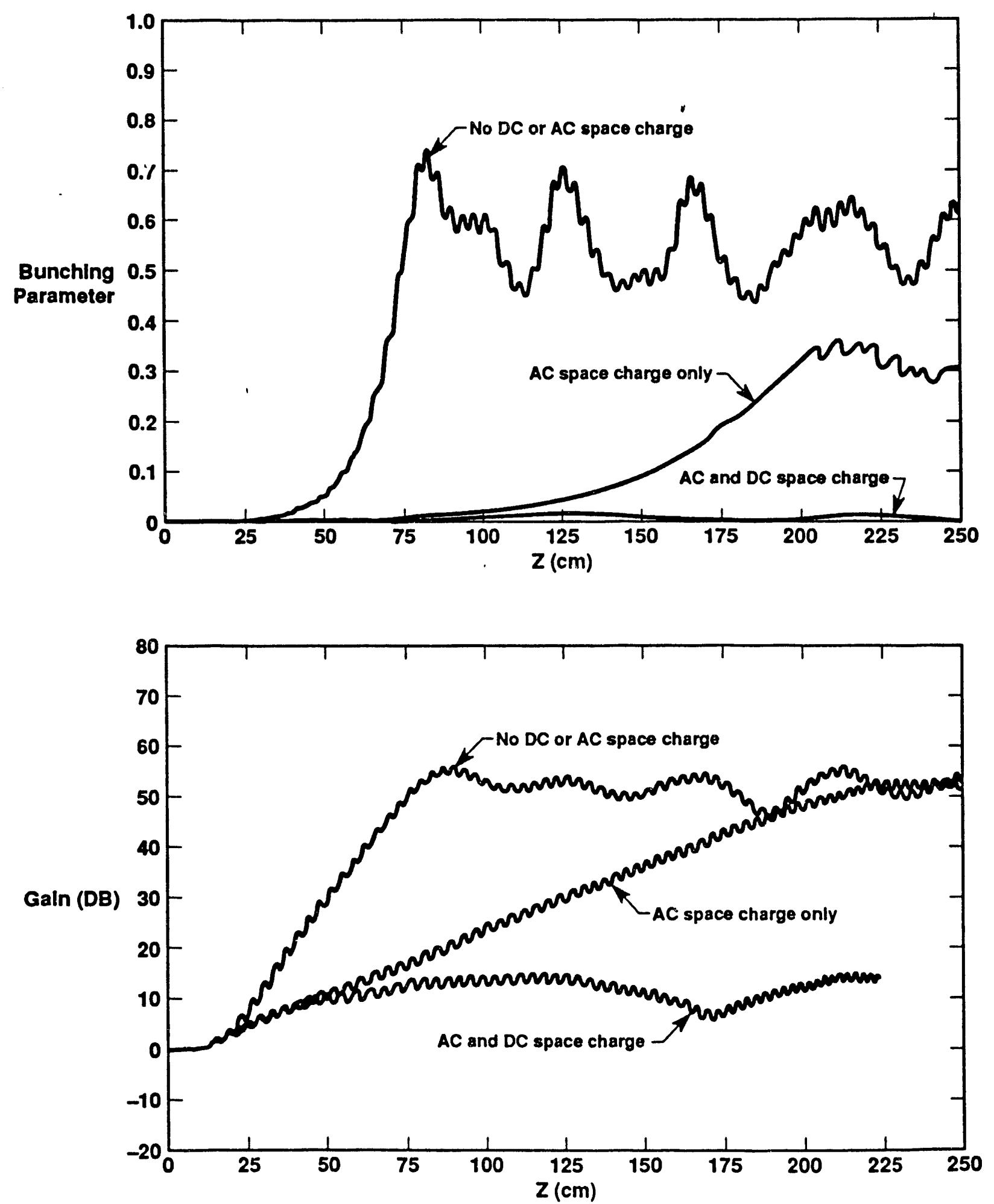

Figure 2a,b 


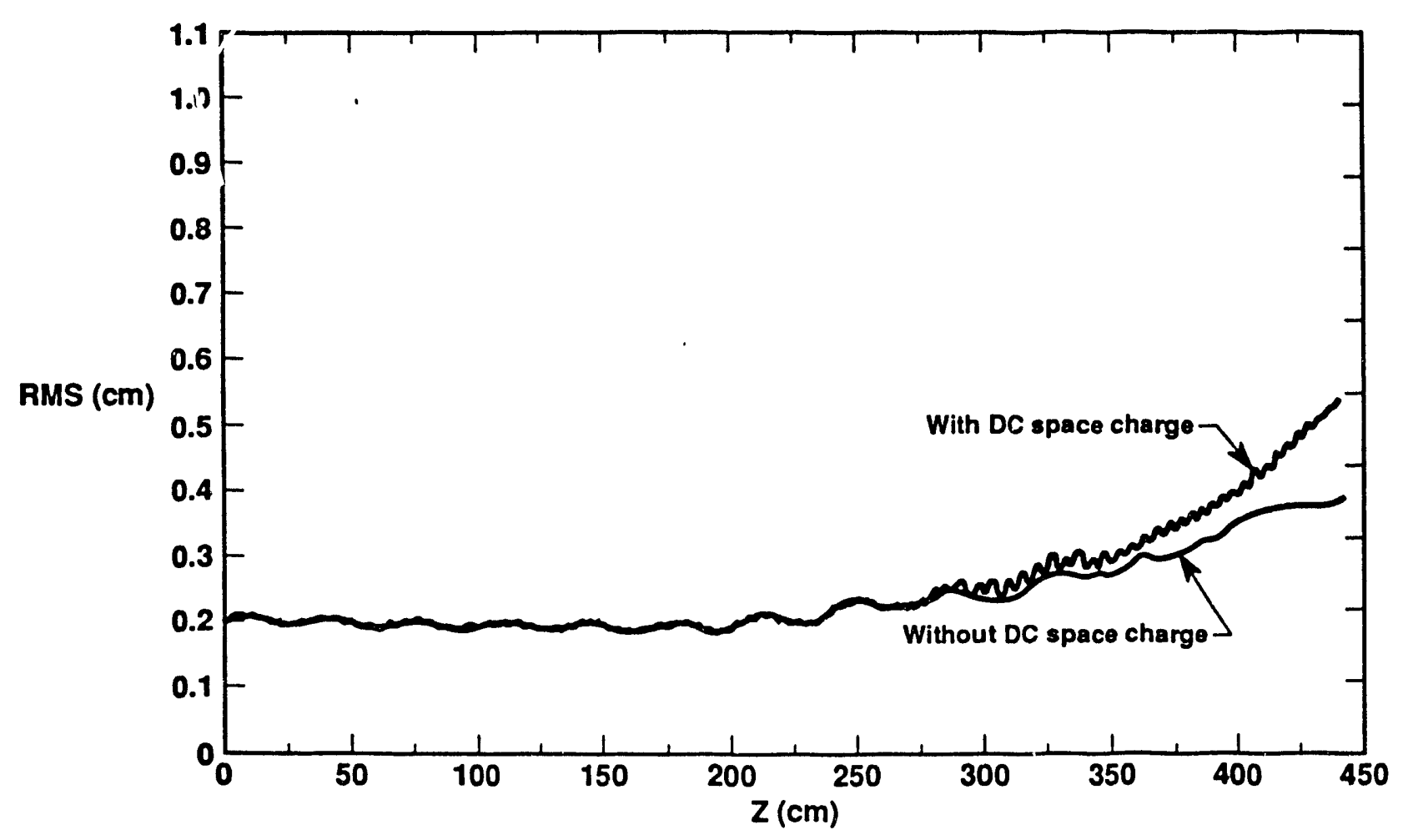

Figure 3 

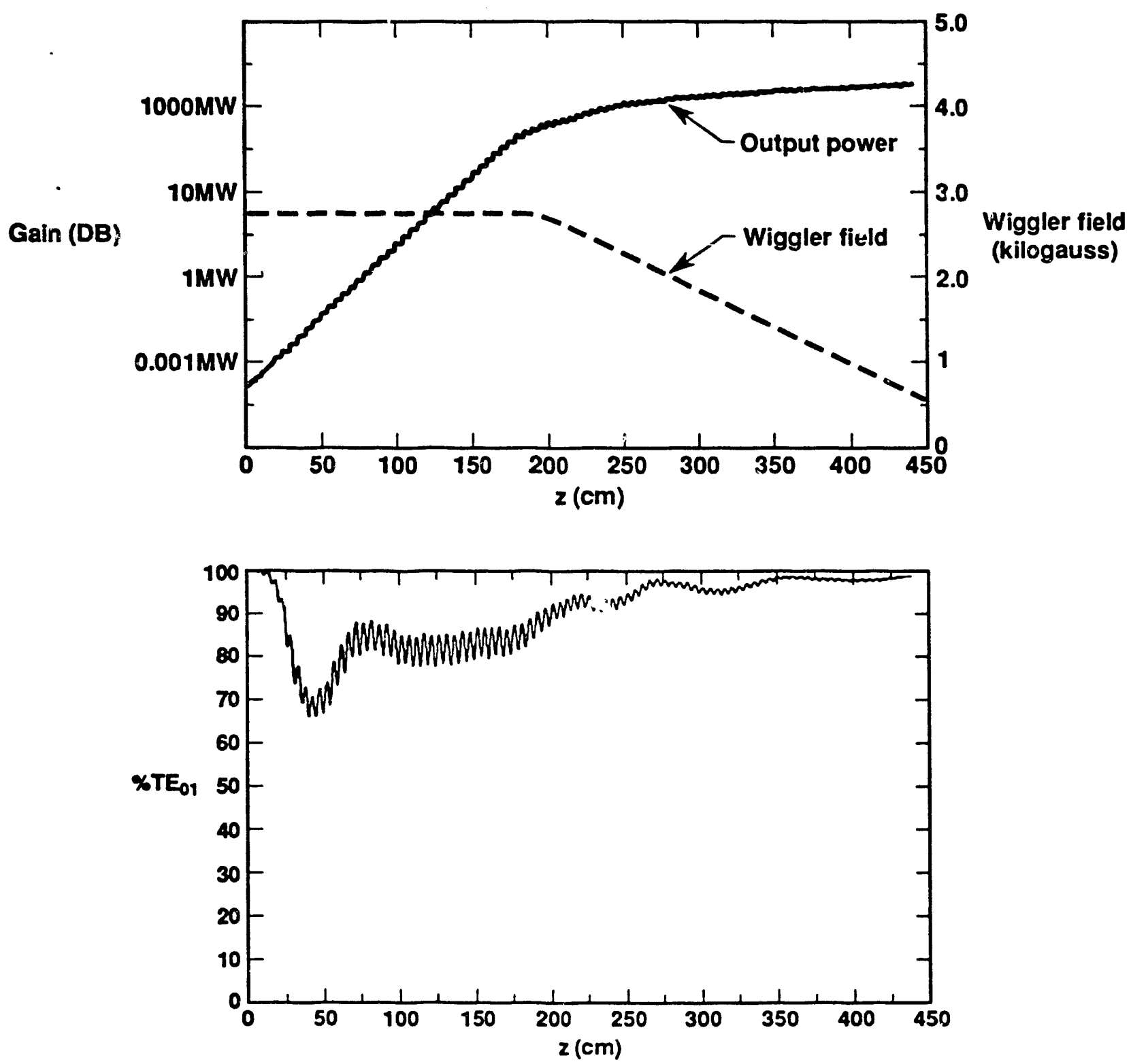

Figure 4 


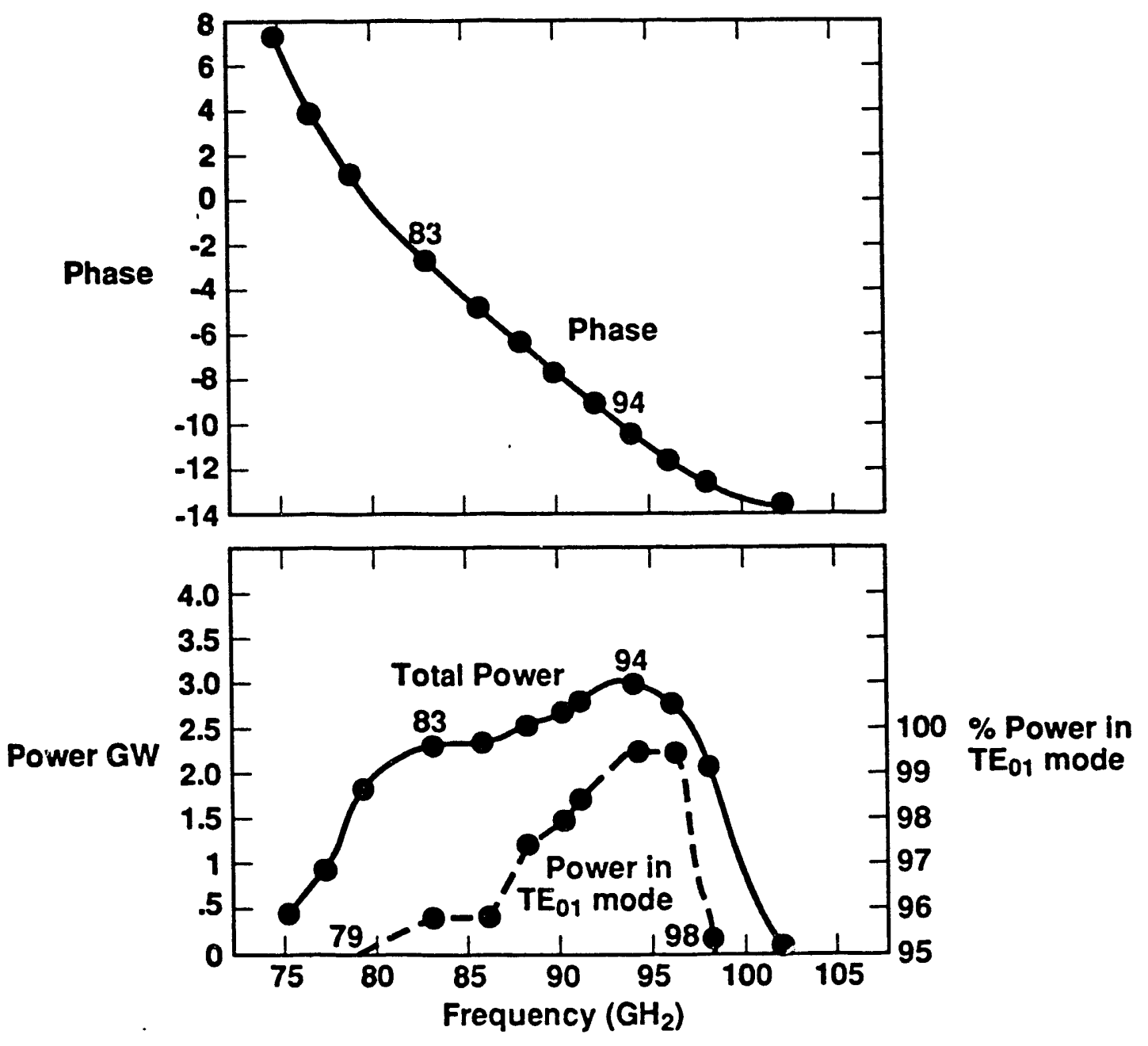

Figure 5 

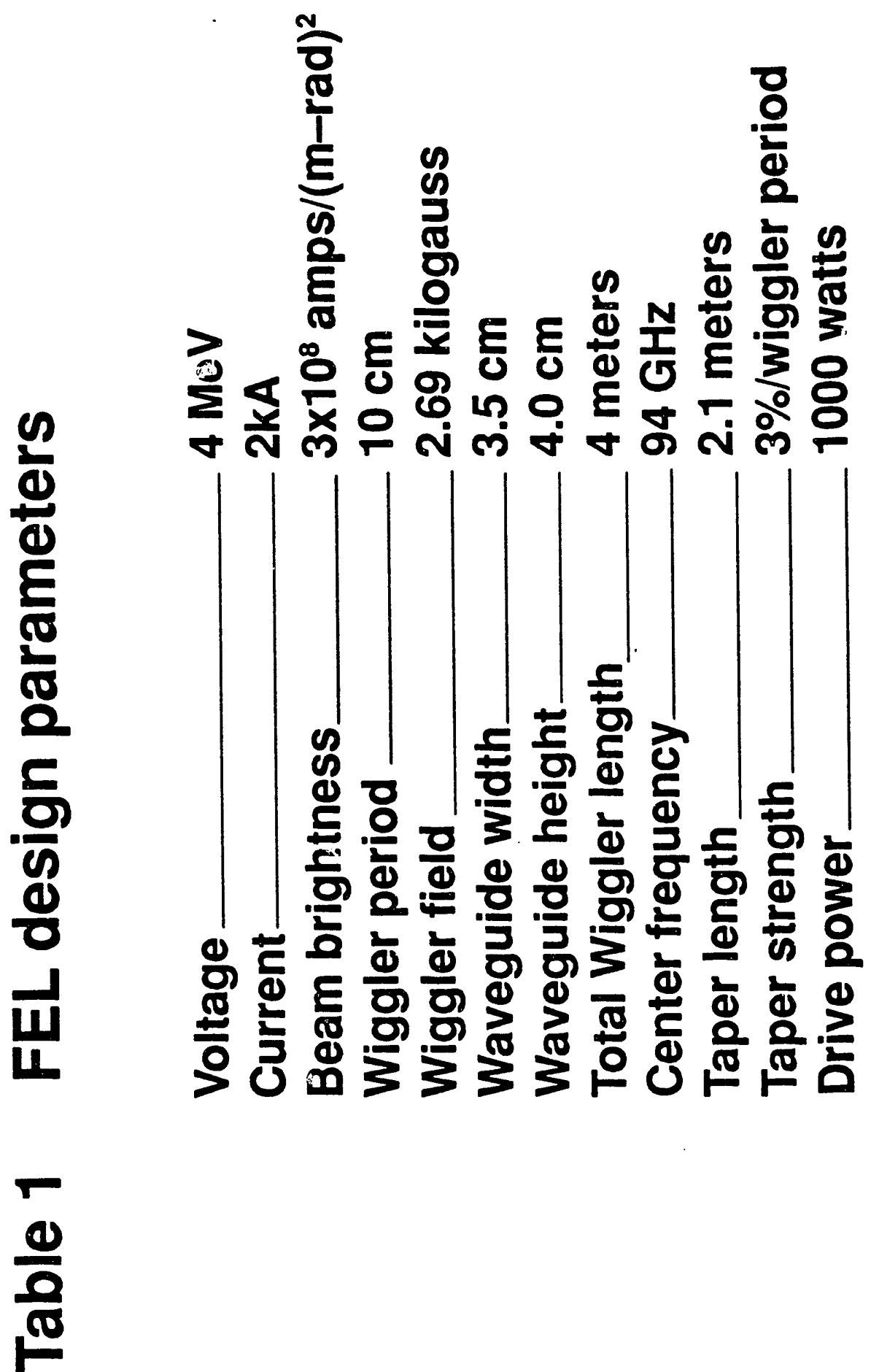

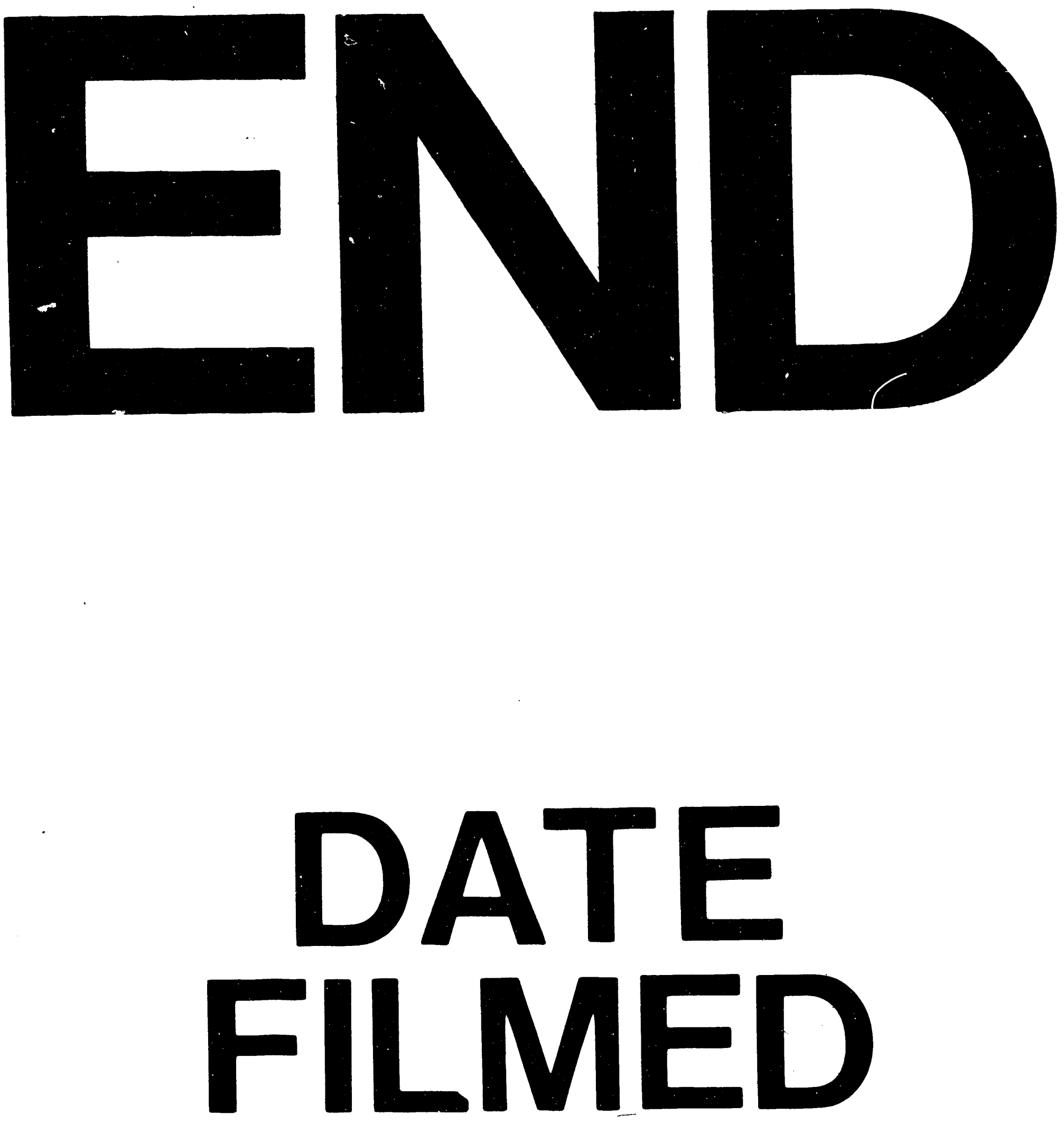

I

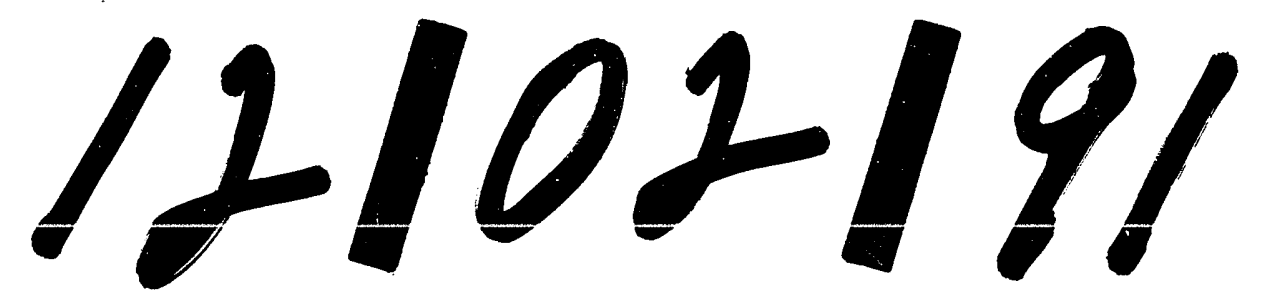

" 


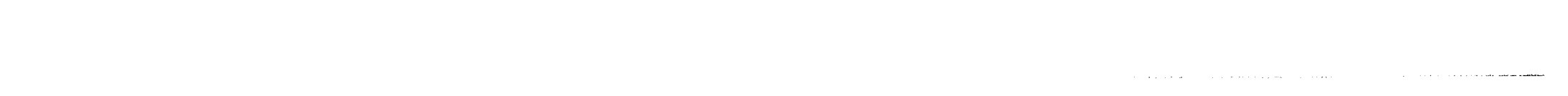

